\title{
La atención selectiva del docente en los procesos de planeación curricular, aprendizaje y evaluación
}

\section{The teacher's selective attention in the curricular planning, learning and evaluation processes}

DOI: $\underline{10.32870 / \text { dse.v0i19.489 }}$

\author{
Óscar Eligio Villanueva Gutiérrez* \\ Leticia Isabel López López ${ }^{* *}$
}

\begin{abstract}
Resumen
La investigación cualitativa realizada tiene como objeto de estudio la atención selectiva —-función mental del ser humano reguladora del flujo de información que llega a la conciencia y otorga el sentido de equilibrio e integridad personal, mediante la selección de estímulos y la relegación de otros, conforme la evaluación del esfuerzo cognitivo- a partir del modelo de Kahneman y el presupuesto teórico de la fenomenología, en relación con las actividades docentes de planificación curricular, aprendizaje y evaluación. Para ello se realizó un estudio de caso intrínseco, en el que se utilizaron dos cuestionarios secuenciales triangulados con entrevista. Participaron 25 profesores neoleoneses de secundarias públicas de una misma zona escolar. El análisis de los datos detecta como arousal (activación), la preponderancia de los contenidos específicos sobre los elementos curriculares en la planificación, con inclusión parcial del enfoque metodológico y la estrategia didáctica correspondiente, disminuyendo las posibilidades de acceder a la competencia, al aprendizaje esperado y al perfil de egreso propuestos institucionalmente.
\end{abstract}

Palabras clave: Mecanismos atencionales - intencionalidad - planeación - modelo Kahneman - fenomenología.

\section{Abstract}

The qualitative research conducted had as its object of study selective attention - a mental function that regulates the flow of information that reaches the conscience and gives human beings a sense of equilibrium and personal wholeness by selecting some stimuli and ignoring others - following Kahneman's model and the theoretical foundations of phenomenology in relation to the teaching activities of curricular planning, learning and evaluation. An intrinsic case study was performed, using two triangulated sequential questionnaires and interviews. 25 teachers from the same public school district in the Mexican state

\footnotetext{
* Filósofo e investigador educativo. Subdirector de Innovación, Investigación y Desarrollo en la Escuela Normal Miguel F. Martínez. Catedrático de Posgrado en la Universidad Pedagógica Nacional, Unidad 19 A, Monterrey, México. ovillanuevagutierrez@gmail.com

** Doctora en Educación. Investigadora educativa. Supervisora en Educación Básica, secundarias. Asesora de Consejos técnicos estatales. Coordinadora estatal de Acompañamiento y Actualización Permanente de Supervisores y Asesores Técnico-pedagógicos en Nuevo León, México. Temas de investigación: Procesos cognitivos. Enseñanza por competencias y modelos educativos. Lenguaje. lety.isabellopezl@gmail. $\underline{\text { com }}$
} 
of Nuevo León participated in the study. The analysis of the data detects as arousal, the preponderance of specific contents over the curricular elements in the planning, with partial inclusion of the methodological approach and the corresponding didactic strategy, decreasing the possibilities of achieving competence, learning outcomes expected, and the exit profile set up by the institution.

Key words: attention mechanisms - intentionality - planning - Kahneman's model - phenomenology.

\section{Introducción}

Lo que Rosselló (2004) define como mecanismos atencionales, activa el núcleo de esta investigación exploratoria en relación con el aprendizaje docente del currículum y la enseñanza orientada a la integración de los saberes generados en el individuo profesor en el marco de la fenomenología. Nos referimos a los aspectos inherentes al aprendizaje, a la enseñanza y a los procesos pedagógico-didácticos que inician en los primeros contactos del docente con un objeto de conocimiento segmentado - en este caso, de conocimiento curricular-, de manera simultánea a sus procesos cognitivos, asociados a la percepción, a la selección de estímulos y al esfuerzo concatenado a la planeación y acción de los procesos de enseñanza y aprendizaje, emergentes de la atención selectiva y sostenida.

\section{Antecedentes}

El mecanismo atencional, como aspecto de la cognición, ha sido rastreado por investigadores de ciencias duras y humanas, de distintas latitudes y tiempos, en búsqueda de certezas respecto al dispositivo de entrada que desencadena el trabajo de la atención docente ante la tarea de gestionar aprendizaje. Rodríguez (2008) aduce que la cognición es la confirmación de que una señal enviada, percibida y atendida, ha sido a su vez interpretada y/o representada por el receptor, lo que nos acerca a la dinámica del proceso cognitivo y permite fundamentar que la ciencia cognitiva es, entre otras cosas, el estudio de la interpretación del contenido simbólico representativo y las respectivas aplicaciones del concepto señal o estímulo dentro del proceso de intercambio e interacción mental.

Sobre la atención como proceso de focalización cognitiva de un sujeto situado en la realidad, Munar, Rosselló y Sánchez (1999) explican que "atender implica seleccionar una parte de la mucha y variada información externa que está disponible en un momento dado en cualquier situación"; señalan que "mediante la atención logramos centrarnos en el análisis de una parte de la información que nos llega a través de nuestros órganos sensoriales"; su conclusión coincide con el planteamiento fenomenológico: "la atención también interviene en la selección de información interna al propio sistema de procesamiento humano, información en forma de conceptos y sus interrelaciones, de imágenes, de planes de acción", en tanto la atención acom- 
paña a la intencionalidad en lo real y en lo mental; asimismo, la perspectiva cognitiva se centra en la importancia de este proceso, el de ir y venir de la realidad a la conciencia y a la inversa: "la atención también puede ejercer la importante función de controlar la actividad cognitiva y conductual que nos permite alcanzar un objetivo" (1999: 34-35). Esto se relaciona directamente con el objeto de estudio de esta investigación, que tiene como propósito explorar su relación con la actividad docente de planeación en la escuela secundaria.

De acuerdo con Rosselló (1994), el mecanismo atencional resultará ser el responsable directo de la organización jerarquizada de los genuinos procesos que, en sentido estricto, tratan y elaboran la información que nos llega desde el mundo circundante y desde ese universo complejo que somos los seres humanos. Argumentamos entonces que el mecanismo atencional que llama la atención de los profesores generará gran parte de la estructura de planeación en el pensamiento docente, dando origen a su definición. Esta postura nuestra la fundamentamos también en Ballesteros (2014: 170), quien considera la atención como el proceso por el cual podemos dirigir nuestros recursos mentales sobre los aspectos más relevantes del medio, o bien, sobre la ejecución de determinadas acciones que consideramos más adecuadas entre las posibles. Para Ballesteros, el estado de observación y de alerta permiten conscientizarse sobre lo que ocurre en el entorno. Consideramos que el estado de alerta de los profesores sitúa los elementos curriculares mediante filtro, en la posición de selección secuencial de elementos.

\section{El contexto educativo. La relación entre mecanismos atencionales docentes en la planificación, enseñanza y evaluación del aprendizaje} El magisterio de nuestra localidad, del país y del mundo, desde el inicio de su formación profesional, requiere conocer y ejercer una competencia docente completa respecto a la precisión de la planeación de una asignatura, incluyendo el uso del tiempo y la innovación curricular, porque la planeación implica incluir la realidad vigente con una intencionalidad retrospectiva y prospectiva; hacia el pasado significa evaluar, y respecto al futuro, implica establecer las finalidades educativas. La importancia de la atención reside en su capacidad de crear este mundo en movimiento como el sustento del aprendizaje para favorecer a los estudiantes en sus rutas y viajes en el aprendizaje de la comprensión, la integración, la aplicación, la evaluación y autorregulación con el aprendizaje permanente significativo, y hacerlo visible; $y$, respecto de los docentes, poder innovar su propia práctica, empleando la atención centrada en el saber experiencial.

Elbaz (1983) define así las categorías del conocimiento práctico docente: conocimiento de sí mismo; conocimiento del medio de enseñanza; conocimiento teórico y procedimental de la materia a enseñar; conocimiento del desarrollo del currículum; y el conocimiento de la instrucción, incluyente del enfoque de enseñanza y de las creencias y teorías sobre el aprendizaje que ha interiorizado el profesor. La dimensión sobre el desarrollo del currículum y el conocimiento de la instrucción inician con los mecanismos atencionales del docente (Martínez, 2004). A su 
vez, Shulman (1987) enuncia las categorías del Conocimiento Base de la Profesión Docente: conocimiento del currículum; conocimiento didáctico general; conocimiento didáctico específico; conocimiento del contenido temático, y conocimiento de los objetivos, finalidades y fundamentos históricos y filosóficos. Las categorías propuestas por ambos investigadores se relacionan con el objeto de estudio de la presente investigación.

En relación con el paradigma creado por Shulman (1987), nos identificamos con la aseveración de que el principal foco de la educación es la práctica, es la unión entre el conocimiento teórico y la acción práctica lo que caracteriza a la educación y requiere de una perspectiva filosófica propia. Nosotros consideramos a los procesos atencionales en esta dirección filosófica, de enlace entre conocimiento práctico y conocimiento base.

Sobre el compromiso profesional del docente de atender las líneas curriculares desde la planeación, Tejada señala que "el profesor formador ha de moverse en el ámbito de la planeación, desarrollo y evaluación de procesos de enseñanza-aprendizaje desde una óptica microdidáctica" (2009: 11-12). La planeación como objeto de análisis tiene dos momentos: desde la responsabilidad de dirigir el proceso de enseñanza es interpretada por el plan de estudios como una realidad digna de reflexión y confrontación cognitiva; y desde la filosofía de la ciencia, como un proceso objeto de validación de saberes por medio de la justificación de hipótesis y las teorías sometidas a recursos de falsación y refutabilidad, que incide en la restructuración de su conocimiento y, de este modo, encontrar en el sustento del paradigma curricular la consistencia, la estructura y la certeza respecto a sus saberes didácticos previos, con evidencia de saber práctico observable. Ambas, forman parte de las exigencias de ampliar las perspectivas de conocimiento sobre la planeación docente.

La SEP define planeación como:

Elemento sustantivo de la práctica docente para potenciar el aprendizaje de los estudiantes hacia el desarrollo de competencias; implica organizar actividades de aprendizaje a partir de distintas formas de trabajo, situaciones didácticas y proyectos, entre otras; deben representar desafíos intelectuales para los estudiantes con el fin de que formulen alternativas de solución (SEP, 2011: 28).

Las implicaciones teóricas de este concepto están en conexión entre lo atencional, desde una teoría sobre la atención selectiva, y lo fenomenológico desde su proyecto de superación de los dualismos a partir de potenciar la atención.

\section{Definición del problema}

¿Por qué la importancia de la atención en los procesos educativos? Los profesores tienen ante sí una realidad compleja que les ofrece una diversidad de elementos estimulantes para convertirse en objetos mentales, para actuar, para transformar. Una cuestión empírica relevante, 
que atañe al valor de la atención para comprender la docencia, está relacionada con la existencia de un conjunto de finalidades prexistentes, otras emergentes, algunas sedimentadas en la profesión docente, que se vuelven argumento teleológico de los procesos educativos. La problemática básica de la atención en la actividad de enseñanza se forma entre un conjunto de realidades; la primera, son las finalidades que circulan en la profesión docente, también las del plan de estudios; finalidades señaladas por la cultura y la gestión institucional. Cada director traduce las necesidades de los procesos de enseñanza-aprendizaje en ciertas normas y reglas de gestión que entrañan sus finalidades.

Ante ello, el profesor está sumergido en sus procesos atencionales de selección de objetos (concretos y abstractos) a distintos plazos, cuya fijación de la mirada para desarrollar el proceso de atención mediante la concentración sugiere problemáticas de definición y decisión cuyo objeto deriva de la finalidad que atiende, cuáles objetos atencionales elige y cuáles sus motivaciones que competen a la continuidad del aprendizaje de cuáles y cuántas asignaturas, por cuál elemento motivacional preservar la atención; desde cuál opción metodológica. Y en un registro real, en tanto correlato de esta deliberación, se encuentran los procesos de aprendizaje, cómo los nombra y qué valor le otorga el docente a la atención en los procesos subjetivos del aprendizaje, cómo jerarquiza sus acciones en su memoria ejecutiva enlazadas a los procesos de atención, cuál camino comprensivo realiza y profundiza con su atención o, si lo desdeña, cómo elabora sus procesos valorativos y morales de acuerdo a la simultaneidad de objetivos de aprendizaje nacionales, internacionales e institucionales de sus alumnos; cómo este desacomodo de la atención crea un correlato con la práctica docente y prefija las rutas de acción y los esquemas cognitivos e interpretativos.

Propiamente, esta es la condición de una planeación que asimila la realidad y reordena el esfuerzo cognitivo centrado en el futuro, desde una fuente de contrastación entre lo real y lo logrado, lo programado y lo logrado, el plazo corto y el largo, los aprendizajes alcanzados y los reservados. Se expresa así la conexión entre lo cognitivo y lo fenomenológico en dos tendencias: aquello que el profesor cree que puede dominar de la actividad docente, y lo otro, que es más difícil de hacerlo. La importancia profesional de la atención reside en que hace emerger un objeto desde una intención e interés particular, adscribiéndola a una finalidad educativa que complemente y supere la conexión con la realidad que plantea el plan de estudios, con su poder de contextualizar.

Este elemento de ontologización de la didáctica y de la pedagogía significa un cambio trascendental en la historia de la actividad docente. La conexión de la pedagogía y la didáctica con la realidad ocurre mediante el replanteamiento de las competencias para la vida, se enlaza la cognición y la valoración con la enseñanza y el aprendizaje con el medio profesional más adecuado, como es la planeación. La planeación representa la programación didáctica y temporal de las acciones, es la actitud prospectiva hacia el tiempo que viene; la evaluación representa 
un instante de detención en el tiempo para mirar y representar el estado y resultado de las acciones en el aprendizaje, es la actitud retrospectiva hacia el tiempo que fue. Sin embargo, la continuidad que requiere la planeación está afectada por la variedad de ámbitos en los que interviene el tiempo, que se viven como una discontinuidad y se asimilan como una carencia del mismo tiempo. La atención selectiva es la condición que permite la activación de la planeación, y el tiempo es su condición de realización.

\section{Preguntas de investigación}

Este reporte de investigación da cuenta de los siguientes cuestionamientos: durante las fases de diseño e implementación de la planificación en las que los profesores emplean mayor carga atencional y esfuerzo cognitivo, ¿qué elementos del currículum y la enseñanza requieren atención? ¿qué elementos de la planeación y la evaluación atiende prioritariamente? ¿qué atrae su atención del plan y programas de estudio? ¿cuáles son los elementos atendidos para llevar al aula la concreción del derecho de todos a aprender?

\section{Objetivos}

Al perfilar la delimitación del objeto de estudio, emerge el objetivo fundamental de este trabajo: descubrir, mediante el diseño e implementación de una investigación exploratoria, las problemáticas de relación entre la atención selectiva docente y la planeación de asignatura, en el contexto de la enseñanza de educación secundaria en una zona escolar. Como objetivos específicos:

- Explorar las categorías de planeación curricular acordes a la atención selectiva de los profesores e identificar las menos atendidas

- Indagar los aspectos de la evaluación que ganan la atención de los profesores.

\section{La construcción teórica de la atención como objeto de estudio en la docencia}

Del concepto de atención destacamos el sentido que le otorga la fenomenología, consistente en una condición que aporta los elementos para el equilibrio y la integridad del ser humano. Esta cualidad de la atención, el día de hoy está puesta a prueba en la actividad docente. Una consideración significativa amerita la referencia a la realidad para ser incluida en las perspectivas didácticas y pedagógicas que prescriben el enfoque y los planes de estudio de la actual reforma, enunciadas en el pronunciamiento "Las competencias para la vida". Los ámbitos de la realidad, la escuela y su contexto, los alumnos y los ámbitos de los enfoques pedagógico-didácticos curriculares, constituyen elementos que se ofrecen a la conciencia que está en un proceso de intencionalidad; ser realista implica confrontar la variedad y amplitud de los estímulos que provienen de ella.

Diálo@os 
La atención es la única cualidad cognitiva que acompaña a la intencionalidad, tanto en lo real como en lo mental; esta nos permite pensar la atención en la actividad docente. Destacamos que la referencia a la atención selectiva compete a una conexión entre atención y realidad con el propósito de controlar el proceso de planear y gestionar la enseñanza, cuyo dilema consiste en si en él tiene cabida también el proceso de aprendizaje, dualidad explícita en los documentos curriculares, e inscrita en el conocimiento práctico docente.

\section{a) La atención en la obra temprana de Ricoeur y su implicación interdisciplinaria}

Ricoeur (1939) considera que la atención tiene un gran valor en el ser humano a partir de tres construcciones en el sujeto: la acción, el conocimiento y la conexión con el mundo. Entonces la atención tiene una participación decisiva en el proceso de construcción de los equilibrios en el sujeto. La atención pone lo que sucede en el mundo como objeto de la intencionalidad, hacia adentro; la atención se adhiere al mundo, su exterior, y su mantenimiento significa la posibilidad del conocimiento. Ricoeur es pionero en la concepción fenomenológica de inscribir la atención como un elemento mediador, plural, con reversibilidad y diversidad de temporalidad, que sucede entre el vaivén mundo-conciencia, y en el interior de la conciencia, entre las diversas facultades, implica una acción de la atención como donadora de realidad y de subjetividad.

La noción ontológica de atención que aporta Ricoeur (1939) brinda la oportunidad de revelar el alcance de la atención en la actividad docente, en tanto que la entiende como una condición que reúne lo objetivo, lo subjetivo, lo inmediato y lo mediato, lo empírico y lo trascendental, la actitud y la voluntad, lo determinado y lo espontáneo, el presente y el futuro. En la perspectiva de la fenomenología, la atención acompaña a los procesos de la intencionalidad. Por ello su acción provee una pluralidad de realidades y de activación de procesos que permiten el flujo y la conservación de las condiciones cognitivas, experienciales, morales, conductuales, lingüísticas y prácticas que ocurren en la profesión docente, aunque el sentido que goza de más consenso es la atención en tanto control cognitivo. Tal y como señalan Posner y Rothbart (2014), la atención es uno de los procesos de control cognitivo claramente relevantes para el desempeño y el aprendizaje escolar infantil (Ilson 2016: 2).

El trabajo del movimiento fenomenológico sobre la atención es atestiguado por Scheler (1987) y Wandelfs (2015), entre otros, que la colocan como componentes de un correlato que incluye los sentimientos, las emociones, la simpatía, la voluntad y la percepción.

Desde la psicología fenomenológica, la atención está considerada como una capacidad relacionada prácticamente con el cerebro, el cuerpo y las emociones. En la filosofía-fenomenología, la actividad atencional en el aprendizaje es un modo concreto de relación del sujeto consigo mismo y con los objetos, identificada por Depraz (2012) en tres gestos: de suspensión, de redirección y de acogida. En esta perspectiva, la atención es el primer objeto implícito de la reflexión. 
b) La atención en las perspectivas teóricas acerca de la educación

El fenómeno de la atención atrae diversas ciencias que investigan al ser humano, algunas son: filosofía, neurociencias, psicología, e incluso, teología. La atención por sí misma representa un desafío para el conocimiento y las filosofías. En el área de los estudios educativos, la observación de la atención se ha ubicado en el perfil de la subjetividad; por lo general, se piensa la subjetividad docente en relación con los procesos deliberativos, y la atención es subsidiaria a ellos (Perrenoud, 2005), procesos argumentativos sin acción definida de la atención (Tardif, 2005), o del pensamiento del profesor (Paquay, 2005). Ellos describen la atención como el encuentro entre la realidad y la conciencia como uno de sus rasgos distintivos, una entidad ligada a los procesos de equilibrio e integración entre enseñanza y aprendizaje, planeación y evaluación.

La intencionalidad está formada por los primeros objetos de la atención; sin ella, los procesos de reflexión no pueden ser constituidos. ¿Por qué la atención de un profesor es atrapada dejándose llevar por los aprendizajes esperados? ¿o por los alumnos de capacidades diferenciadas? $\mathrm{O}$ al contrario, ¿por qué se distrae el profesor o no sostiene su atención respecto a los procesos de la enseñanza?

Desde el método fenomenológico, la atención centrada en la práctica también posee una dualidad elemental; por una parte está la focalización de la atención, delimitada y centrada, y por la otra está como una cualidad abierta y panorámica. En la dimensión de la clase, la primera se relaciona con la actividad en sí misma; y la segunda, con la posibilidad de respuesta del alumno a la tarea, centrada en el cumplimiento con el aprendizaje esperado.

Para dar un sentido científico a esta propuesta ontológica, en un primer momento entendemos que está creándose un proceso de interdisciplinaridad apuntalado por la fenomenología y reencontrado por las ciencias cognitivas, la neurobiología y las ciencias experimentales, con intersecciones estabilizadas acerca del reconocimiento de la atención. Por tanto, apuntamos que la atención es una promesa de superación de los dualismos, tan caros a la epistemología y la ontología de las ciencias humanas y sociales que definen la llustración y la Modernidad.

Como vertiente intersubjetiva de la atención, existe un segundo momento: atender a sí y al otro, el cuidado de sí mismo y la preocupación por el otro, que constituye una práctica relacional que define a la procedimentalidad atencional (Depraz, 2012: 27). Las profesiones dedicadas al cuidado del otro, como la enfermería, la psicología, el trabajo social y la docencia, tienen como igualdad ontológica este matiz de la atención como cuidado del otro.

Un tercer momento reside en la conexión con el sentido común, que se asume como verdad natural en la vida común; en el fenomenología es nombrada por Pereira (2015) como la "ingenuidad natural de nuestra vivencia introspectiva". En este proceso la cultura predomina en el sentido común. Instalado en el mundo de la vida, el alumno será conducido al mundo del saber; para realizar este tránsito se requiere que el profesor previamente supere el mundo de la vida y el mundo cotidiano. Si el enfoque por competencias para la vida implica una conexión con ella 
y su mundo, cómo incluirlo entonces es cuestión pedagógica y didáctica, y cómo superarlo es cuestión didáctica, pedagógica y filosófica; esto se centra en el conocimiento práctico.

Un cuarto momento consta de las relaciones cognitivas entre la atención, la memoria, el razonamiento y la percepción, que definen a los estudios cognitivos y que se centran en el conocimiento teórico y conceptual en la esfera del conocimiento teórico. Así, mediante la atención es posible replantear el debate entre conocimiento práctico y conceptual-teórico. ¿Cómo aprehender la profesión docente desde el análisis de los procesos atencionales?

Señalamos enseguida las dimensiones teóricas fundamentales de la atención en la docencia constituida como un objeto de estudio.

\section{c) Indagación conceptual}

De modo delimitado, este apartado se sujeta a la atención selectiva. Las investigaciones sobre la atención confirman la advertencia de Rosselló (1999) respecto a la dificultad de definirla aún en los dos últimos siglos: proceso de naturaleza compleja y diversa, con una comprensión solamente parcial de su funcionamiento (Alameda, 2004: 5). Pío Tudela (1992; 2015) la definió como el "mecanismo central de capacidad limitada que controla y orienta la actividad consciente en función de un foco determinado"; y Tejero (1999), como un "mecanismo cognitivo de control voluntario sobre la actividad cognitiva, en el sentido de activar, inhibir y organizar las operaciones mentales cuando éstas no pueden desarrollarse automáticamente" (Alameda 2004: 6).

Considerando los aportes teóricos de Kahneman (1973; 1997), Vega (1984), Tudela (1992), García-Sevilla (1997) y de Rosselló (1997), Alameda (2004) la definió como la habilidad compuesta por estrategias para la optimización del funcionamiento del sistema cognitivo, y como capacidad de procesamiento, capacidad que puede distribuirse a diferentes focos de manera variada también mecanismo activador de procesos. Para Rosselló (1997) se trata del mecanismo responsable de la organización jerarquizada de los procesos que abordan y elaboran la información llegada del mundo circundante y desde el universo complejo que constituye el ser humano (Añaños, 2002).

A finales del siglo IXX, James la definió como "el proceso por el que la mente toma posesión, de forma vívida y clara, de uno de los diversos objetos o trenes de pensamiento que aparecen simultáneamente". Focalización y concentración de la conciencia son su esencia. Implica la retirada del pensamiento de varias cosas para enfocarse en otras (1890: 403).

Fuenmayor y Villasmil (2008) señalan que la atención surge cuando el sujeto empieza a captar información por medio de los sentidos, y comienza a advertir cualidades en todo o en una parte, debido a que se divide su atención y puede hacer más de una cosa simultáneamente; señalan que se adquieren destrezas y se desarrollan rutinas automáticas para realizar una serie de tareas sin mucha atención. Este es el principio de la teoría de la capacidad de Banyard 
(1995), que se refiere a cuánta atención se puede prestar en un momento determinado y cómo esta puede cambiar dependiendo de la motivación del momento. A partir de este enfoque, nos referimos a una atención selectiva, porque a lo largo de toda su vida el hombre selecciona e interpreta continuamente la información que recibe del medio circundante (Fuenmayor y Villasmil, 2008: 8).

Alameda precisa: "los procesos de atención focalizada son aquellos en que los organismos atienden selectivamente a un estímulo, o algún aspecto del mismo con preferencia sobre otros estímulos" (2004: 9-10). Menciona dos interpretaciones de esto: la que posibilita el análisis más adecuado de la información relevante, siendo el principal mecanismo atencional el procesamiento selectivo, y la que posibilita la expresión de la respuesta más adecuada.

Kahneman $(1973 ; 1997)$ reafirma que la atención implica la existencia de un control del organismo sobre de la elección de los estímulos que, a su vez, controlarán su conducta dado que la atención es algo más que una mera selección al relacionarse también con la cantidad o la intensidad. Este investigador considera que, tanto con la selección voluntaria como con la involuntaria, hay que tener en cuenta los aspectos intensivos de la atención (Añaños, 2002).

\section{d) El modelo teórico de Kahneman en la atención docente selectiva y limitada}

Nosotros consideramos que el modelo teórico de Kahneman (1973) apoya la interpretación de los procesos de atención focalizada de los profesores respecto a los elementos y fases de la planeación de asignaturas, por sus características:

- El modelo de recursos simples o centrales es el primero de capacidad de atención limitada. La dificultad de la tarea controla la capacidad de procesamiento; si una tarea es compleja, ocupa más recursos del sistema; es el sistema cognitivo el que realiza el trabajo o reparto de recursos atencionales. El reconocimiento de la dificultad y complejidad de la tarea se refiere a los componentes involucrados en la actividad docente, por lo que atañe a la conexión de la atención en dos vertientes: en su relación con la subjetividad y con la realidad; el reconocer una dificultad implica disminuir los recursos atencionales para el sistema cognitivo.

- La evaluación de la demanda de capacidad para establecer el consumo de recursos atencionales en cada tarea o proceso, establece la relación entre capacidad de atender y el esfuerzo; trata los aspectos selectivos de la atención, y también los aspectos intensivos. Transitar de los contenidos a los aprendizajes esperados es una cuestión que se traduce como una correspondencia entre la capacidad de atender y el esfuerzo. El aumento de la capacidad es posible por una actividad hermenéutica, al asumir los aprendizajes esperados como una totalidad organizada desde sus verbos de desempeño (López, 2015; Villanueva, 2010). 
- Los estímulos pasan a un registro sensorial. A partir de este registro se produciría un filtro de selección antes del análisis perceptivo. Después de este, se produciría la respuesta. La atención sería antes de la percepción.

- Ofrece una solución a la sobresaturación de estímulos a atender, considerando que los recursos de procesamiento son limitados: la atención como recurso limitado asignado a las tareas con flexiblilidad; dedicamos una parte de la atención a una tarea y otra parte de la atención a otra. En la fase de diseño e implementación de la planeación, los profesores seleccionan algunos elementos estimulantes, relegando otros también importantes.

\section{Enfoque metodológico}

Se seleccionó el estudio de caso por la necesidad de observar un fenómeno particular que revelara la relación que se encuentra entre la actividad de enseñanza, la atención selectiva del docente en los procesos de planificación, aprendizaje y evaluación, y en consecuencia, estar en posibilidad de realizar aportaciones pertinentes atendiendo lo que el estudio revela. Acorde con Stake(2005), por su finalidad, se trata de un estudio de caso intrínseco debido a sus especificaciones de valor propio a favor de alcanzar la comprensión del caso a estudiar por el interés y relevancia de la temática. La elaboración de la metodología parte del paradigma cualitativo, en el cual el uso del cuestionario es considerado válido en una investigación exploratoria, como Rodríguez, Flores y García lo suscriben (1999: 185). Por ello, para la recolección de los datos se emplearon dos cuestionarios secuenciales de preguntas abiertas, lo que Álvarez-Jurgenson (2003: 148-151) denomina método híbrido. El primer cuestionario consta de tres preguntas abiertas, del cual emergieron y se identificaron las unidades de análisis; el segundo consta de ocho preguntas, del cual surgieron y se trabajaron las categorías teóricas en torno a las selecciones atencionales de los profesores respecto a la enseñanza, la planeación y la evaluación. Los cuestionarios se aplicaron a 25 profesores de secundarias públicas vespertinas de una zona escolar urbana, con una distancia temporal de seis semanas. Cinco maestros participaron en la entrevista, instrumento mediante el cual se triangularon los datos obtenidos de los cuestionarios.

El tratamiento de los datos procedió de identificar y clasificar los elementos que incluyen las unidades más relevantes y significativas. La información obtenida aparece compartiendo significados importantes focalizados en los profesores participantes. De allí emergieron núcleos temáticos variados.

\section{Desarrollo}

La presente investigación busca reconocer cuáles elementos curriculares representan un foco de atención para los profesores al momento de trabajar la planeación de su asignatura, y comprender la dinámica que implica. Atañe al análisis de la contribución específica lo que los pro- 
fesores participantes en esta investigación realizan en las aulas respecto a la atención selectiva aplicada sobre los planes y programas de estudio, en el entendido de que del esfuerzo cognitivo vertido en estos aspectos surgen condiciones de conocimiento y de guía para su acción docente, y se posibilitan las reflexiones y consideraciones pertinentes en función de los hallazgos y resultados sobre cómo los elementos curriculares que captan su atención, se significan en distintas posibilidades y frecuencias de su actividad de enseñanza y los aprendizajes significativos resultantes.

Reargumentamos: en la actual situación histórica de la docencia, la capacidad humana más cuestionada en esta sociedad posmoderna y con más posibilidades de socavar o producir los procesos de integración, está en los procesos atencionales del profesor. En la cotidianeidad escolar se requiere atención para echar a andar el proceso de enseñanza-aprendizaje y mantenerlo; la formación docente combina la atención focalizada y la dividida, resultando dilemático el mantenimiento de la atención en procesos abstractos y concretos; durante la incorporación profesional de los docentes, la condición atencional inmersa en el choque de realidad se dispersa, su restructuración define el término del periodo de incorporación en los primeros meses (Villanueva, 2010). La importancia de la noción de atención puede valorarse al considerarla como el principio pedagógico primero en educación básica y normal.

\section{Análisis de resultados}

El análisis cualitativo de los datos muestra la relación entre la atención selectiva del docente respecto a los tres procesos investigados: enseñanza, planeación y evaluación.

\section{La atención selectiva en el aprendizaje y la planificación. El foco curricular}

En los procesos de planeación, la atención del docente se orienta hacia los contenidos específicos; es en lo que se distingue la intervención de la atención selectiva. Además de esta elección u orientación, también se presenta la de recurrir —en menor proporción- a los aprendizajes esperados y las competencias disciplinares. De esta manera, de la atención selectiva se transita a una experiencia de amplitud atencional. Pero existe una tendencia más: la vertiente de la atención selectiva se encuentra en la conexión entre competencias generales y estrategias didácticas. Ambas tendencias comparten centrarse en el eje concreto-abstracto, lo concreto de los contenidos y las estrategias, y lo abstracto de las competencias disciplinares y generales. Ambas presencias muestran la falta de atención profunda a los aprendizajes esperados, o bien al tipo de atención que interviene en los aprendizajes esperados y la gama de finalidades curriculares, propósitos, objetivos, estándares curriculares. Entre la atención selectiva y las finalidades curriculares se requiere algún tipo de propuesta de conexión didáctica y pedagógica. Mientras tanto, las finalidades y los contenidos son opciones incompatibles o excluyentes, como señala un profesor: "al enfoque nos remitimos cuando dudamos, la experiencia nos señala las posibilidades de mejorar la enseñanza..." (Profr. de Ciencias II).

Diólo@os sobre Educación año 10 | número 19 | julio-diciembre 2019 | ISSN 2007-2171 
Al elaborar la planeación, la preminencia de atender los contenidos específicos sobre otros elementos del currículum (aprendizajes esperados) conlleva diversas intenciones, a favor y no, del logro de los propósitos curriculares y disciplinares; denota claridad en la demanda estudiantil por mejores prácticas de enseñanza y, simultáneamente, la desviación de la atención en el nivel taxonómico que indica el aprendizaje esperado, principio y fin de las actividades de enseñanza en favor del desarrollo estudiantil planteado. “La comprensión de los temas, la redacción y la aplicación del aprendizaje son procesos que requieren incremento de atención como docente" (Profra. de Español III). Encontrando el sentido de los datos, el desarrollo del currículum se activa por la atención selectiva del docente en el conocimiento del contenido, se encuentra disminuida la atención en los aprendizajes esperados en relación con la metodología.

\section{El eje transversal: la metodología}

Un hallazgo fundamental de la investigación es el referente a la falta de conección e integración de las líneas curriculares en las que la planeación se fundamenta, con la metodología de enseñanza y la medición de los logros del aprendizaje; esto es, con el proceso de evaluación. Las reformas curriculares 2006 y 2011 promueven la enseñanza por competencias, y después de una década aún persiste la falta de atención a la integración, y como dificultad —-podríamos afirmar-, la inexistencia de la evaluación consistente de competencias desde las prácticas cotidianas de coevaluación, heteroevaluación, autoevaluación, de la evaluación formativa, cualitativa y cuantitativa; "la evaluación formativa capta mi atención regularmente, pero reconozco que todo el proceso de aprendizaje requiere evaluación" (Profra. de Historia I).

\section{Planeación, aprendizaje y evaluación}

Entendiendo como una continuidad de la planeación a estas tres actividades, y la posibilidad del enlace con la atención, alcanza a apreciarse que los profesores sí la distinguen y se manifiestan a favor de las actividades de proyectos, otras que presuponen explicaciones de procesos y en las que interviene la tecnología. Una mayor cantidad de ellos distingue el trabajo en equipo, el colaborativo. Se encuentra que en el marco de atención a la planeación sí hay distinciones de la evaluación; sin embargo, las experiencias de la evaluación únicamente remiten a la observación, por parte de los maestros, de un aumento de uso de la atención en esta y de mayor claridad en los criterios para evaluar. Este tránsito retroactivo de la evaluación a la planeación resulta más atribuido al enfoque de competencias: "la evaluación por competencias requiere de mejor atención de parte del docente", afirma una profesora de Química. Aunque es necesario registrar que desde las respuestas de los docentes, el proceso de evaluación carece de una conexión con los principios de integración. En la evaluación formativa sí existe una clara tendencia hacia la dimensión del aprendizaje. En este aspecto, los profesores presuponen que la cuestión se trata o está unida a un incremento de atención, como lo refiere otra maestra de ciencias; ella 
sugiere un uso con incremento de atención desde los inicios de cómo planear y evaluar cierto aprendizaje esperado. En este aspecto, consideramos que así la evaluación formativa puede representar un potencial para transitar de la evaluación de contenidos y actividades hacia la reflexión de la integración, que significa un uso cualitativo de la atención. En los datos aparecen escasos pronunciamientos por los aprendizajes esperados. Creemos que una evaluación formativa, organizada desde la atención e interpretación de criterios de evaluación sobre los aprendizajes esperados, puede poseer aproximaciones valiosas a la integración de los aprendizajes.

\section{Atención selectiva del docente y aprendizaje estudiantil}

Respecto al aprendizaje, surge una tendencia de la intencionalidad hacia lo abstracto de los niveles del pensamiento: comprensión, interpretación, reflexión y análisis, y por otra parte, aunado a la participación y disposición, interés, motivación y curiosidad de los alumnos, se revela como indispensable para que ocurra el aprendizaje, desde la perspectiva de los profesores. Los datos revelan que los niveles de metacognición y evaluación tendientes al ejercicio de la autorregulación en el aprendizaje, requieren afianzar los bucles que demanda la metodología curricular: es necesario el uso de la comprensión y el análisis en los aprendizajes esperados rumbo a su aplicación, para darle significado al aprendizaje; además, condición indispensable del pensamiento reflexivo, creativo y generador de hábitos de pensamiento productivos y conciencia de estos en los docentes, y estos como modeladores de aprendizaje para estudiantes, rumbo a los niveles de síntesis y evaluación, tanto de motivaciones como de logros y eficiencia en los aprendizajes.

Este resultado promueve el pensamiento propositivo al considerar cómo convertir esta tendencia de abstracción en su traducción didáctica a conocimiento práctico y a valorar la intervención de la evaluación. Este aspecto hermenéutico, cognitivo y motivacional tiene un lugar en las creencias que dominan al docente, las atiende de modo panorámico. Sin embargo, en la atención selectiva los profesores advierten la importancia de fortalecer las estrategias didácticas a la par del enfoque, así como estar en posibilidad de participar en redes de conocimiento profesional mediante las tecnologías de información y comunicación (TIC).

\section{Población estudiantil heterogénea y atención docente}

La tendencia de intencionalidad en el desarrollo cognitivo de los alumnos presenta una ruptura, justificada plenamente por el derecho de todos los alumnos a aprender en un marco de equidad e inclusión educativa en el cual, por supuesto, dentro de una población estudiantil heterogénea se encuentran los alumnos con necesidades diferenciadas, y que se presenta con un porcentaje significativo en las escuelas secundarias de los profesores participantes; estos señalan que los alumnos captan su atención por la necesidad específica, que buscan cubrir con unos minutos de atención individualizada, adecuando la planeación y empleando preguntas genera- 
doras de interacción, materiales audiovisuales seleccionados para ellos, buscando su confianza, a la par de apoyarse con el colegiado docente; "procuro brindarles atención personalizada al menos unos minutos de la clase, con estrategias diversificadas, muchos de ellos tienen estilo de aprendizaje visual y preparo imágenes y presentaciones power point para captar su atención, con adecuaciones a los contenidos y a las actividades" (Profra. de Español I). Sin embargo, la función tutorial aparece, pero no acompañada de la consejería de la Unidad de Servicios de Apoyo a la Educación Regular (USAER) u otra institución que brinde certezas en las interacciones escolarizadas de los profesores con los alumnos. Es el profesor que, con la atención individualizada como estrategia de solución para los niños de capacidades diferenciadas, expresa la validez de la atención selectiva.

\section{El tiempo: recurso vital en la docencia}

La organización del tiempo y el conocimiento de los alumnos surge en trazos puesto que se diluye la atención de los profesores, como expresión de una ausencia de dominio sobre él y, a la vez, de la carencia de modalidades de certidumbre sobre su uso, mientras que el conocimiento sobre los alumnos implica un uso de todos los tipos de atención, así como un uso intensivo y de amplia duración. Esto es referido como algo importante, sin embargo, crece la incertidumbre puesto que en la organización y uso del tiempo no se aplican los principios de la enseñanza por competencias, el aprendizaje cooperativo y los trabajos en equipo; "mejorar la competencia comunicativa en la producción de textos escritos y orales de mis alumnos, a la par de lograr mejor desarrollo y competencia lectora, la comprensión específicamente ocupa cantidad significativa del tiempo escolar" (Profra. de Inglés I, II y III).

Los profesores también reconocen que a cada acto nuevo corresponde agregar tiempo cronológico y crear un nuevo sentido cualitativo del uso del tiempo, lo que implica una concepción vivida diferente, tanto de la atención como de la cognición; "los resultados de los alumnos, sus calificaciones en cada bloque y las evaluaciones bimestrales merecen más atención, no dejarlos pasar al iniciar cada bimestre, sino trabajar el sentido de estos datos" (Profr. de Formación Cívica y Ética II). Esta tendencia de una evaluación individualizada sí se vislumbra. Planear considerando los resultados de la evaluación, surge con un índice de atención centrado en los contenidos y en los aprendizajes esperados, interpretado por el conocimiento experiencial.

\section{La atención selectiva en el proceso de evaluación}

La mayor carga de esfuerzo subjetivo y cognitivo docente está en el conocimiento de la dimensión curricular. En sus vertientes de condición comprensiva, hermenéutica, cognitiva y conceptual del programa, destacan de modo elemental tres temáticas: la transferencia de conocimientos acerca de contenidos de aprendizaje a la actividad de evaluar; la elaboración de medidas prácticas para la aplicación del concepto de transversalidad; y el orden de las actividades para hacerlo realizable y así 
alcanzar el perfil de egreso. Un segundo nivel de la atención selectiva está puesto en la pertinencia y eficiencia de este proceso para comprenderlo. La evaluación, desde la retrospectiva, implica hacer visibles las etapas del proceso de transversalidad, que supone una nueva faceta de la conexión entre lo concreto de un producto y lo abstracto de un proceso. Si bien la evaluación resultó ser una dimensión de bajo nivel de atención selectiva comparada con las necesidades comprensivas y conceptuales acerca del programa, en el momento de la planeación sí se identifican las actividades recurrentes de evaluación y alcanzan prioridad las modalidades de la evaluación, la participación, tareas y trabajos de los alumnos, la atención en los aprendizajes esperados; queda así la constancia entre lo concreto y a corto plazo del aprendizaje, con lo abstracto y a largo plazo. Con menor importancia y con atención limitada figuran la evaluación formativa y el logro del perfil reflexivo; y con menor presencia, la autoevaluación. Sin embargo, los docentes aseguran la importancia de los logros y los procesos de aprendizaje individuales para considerar la evaluación con sentido profesional pleno.

\section{Discusión y conclusiones}

Con esta investigación exploratoria abrimos la discusión en dos frentes: uno, hacia la referencia de la atención respecto a la teoría de la toma de conciencia de Perrenoud (2005) y la teoría del actor racional de Tardif y Gauthier (2005); y el otro, respecto a la importancia de distinguir y recolocar las nuevas vertientes dualistas que acompañan al reciente discurso de la reforma educativa (Paquay, 2005).

La comprensión de la actividad docente, al centrarse en la atención, logra articular un conjunto de opuestos, pares oposicionales o "dobles". Cuestiones en las que está centrado el debate filosófico sobre la constitución actual de la pedagogía como ciencia. Destacan: lo concreto-empírico y lo abstracto; lo inmediato en el corto plazo y lo mediato a largo plazo; lo objetivo de lo real y lo subjetivo de lo imaginario; y lo simbólico, aislado y junto, parte y todo, medio y fin, producto y proceso, inmanente y trascendental, continuidad y discontinuidad, límite y alcance, lo cuantitativo y lo cualitativo, problema y solución. En esta realidad, la atención revela su alcance de importancia epistémica, ontológica, intersubjetiva y curricular; reside en su poder de mediación, que Ricoeur, en su artículo "Las conexiones filosóficas de la atención" (1939), pone de manifiesto con su argumentación.

Emergen dos tendencias de articulación entre la teoría y la práctica, mediadas por la atención. Una: de los conceptos e intereses abstractos a la experiencia empírica y de esta, en tanto conocimiento práctico hacia la teoría. Se revelan como realidades más allá de la superficie y la apariencia -y por lo mismo, lejanas a toda instancia de control y poder sobre ellas - cuestiones como la urgencia del conocimiento de los alumnos, la identificación de los criterios de evaluación, la intervención del tiempo institucional en combinación con el tiempo psicológico del aprendizaje. Aunque existen tendencias de asombro e interés por los procesos de abstracción (comprensión, inter- 
pretación, reflexión, análisis), está ausente una articulación con las realidades más complejas, mencionadas al principio de este párrafo.

La otra: desde el análisis cualitativo de los datos, se evidencia que los profesores hacen uso de la atención selectiva para analizar el discurso curricular y comprenderlo, lo que no significa que todos los elementos del documento oficial emitido por la SEP para dirigir el proceso de aprendizaje de calidad en las escuelas secundarias sean adecuadamente atendidos. Se trata de una conquista voluntariosa de cada docente —y se sugiere-, secuencial, para ofrecer mejor planeación, mejores oportunidades de aprendizaje, mejor enseñanza, mejores prácticas de evaluación y, en consecuencia, mejorar los logros educativos escolares; están comprometidas varias competencias docentes en ello: organización, conocimiento práctico, conocimiento curricular, conocimiento didáctico general y específico, creatividad en los procesos de enseñanza y en la innovación, y dominio del proceso de la evaluación integral con enfoque formativo.

Perrenoud (2004) identifica diez competencias profesionales del docente. En esta discusión referimos la necesidad de replantear tres: organizar y animar situaciones de aprendizaje; gestionar la progresión de los aprendizajes; y organizar la propia formación continua. Es importante establecer una conclusión acerca de los contenidos de enseñanza y los aprendizajes esperados en la actividad de la planeación. Los aprendizajes esperados constituyen la guía de la intención didáctica y, a la vez, un eje pedagógico generado por la atención selectiva, mientras que los contenidos de enseñanza constituyen una estructura de base para el desarrollo de la actividad de enseñanza. Este eje es una relación que nace en la planeación y adquiere correlación y luego duración, pues los aprendizajes esperados se vuelven un referente para la actividad de la evaluación. Para la comprensión de este fenómeno, concluimos en la supremacía de los aprendizajes esperados ante la necesidad de los profesores de tener una guía, y este fenómeno se realiza tanto en la planeación como en la evaluación, centrados en la atención selectiva.

La distancia colocada entre la cognición de los docentes y los elementos que les representan retos, y la demanda de mayor capacidad para integrar los elementos curriculares en su planeación y práctica docente, despliega altas y bajas en la consecución del equilibrio entre el ideal y la realidad educativa planteada. Esta es una respuesta de los profesores ante los estímulos curriculares que captaron su atención.

Concretar los fines de la enseñanza requiere el dominio de dos ejes pedagógicos: didáctica y organización, y gestión de aprendizajes. ¿Cómo concatenar los procesos atencionales sin riesgo de dispersión de esfuerzos? ¿Cómo debe ser el proceso docente de autoreflexión y reconstrucción del conocimiento pedagógico-curricular rumbo a la pedagogía de la integración con los conceptos clave correspondientes de transversalidad, competencia y eficacia? Este es el aspecto medular de la atención del profesor y los filtros del esfuerzo cognitivo para reorientar los procesos de dominio y la intencionalidad interpretativa en la implementación, en la heterogeneidad de condiciones y poblaciones. Consideramos que la atención selectiva 
deviene como el paradigma predominante de la atención en la actividad docente en la educación secundaria.

La dificultad de hacer compatibles el enfoque didáctico, las actividades de enseñanza y los referentes teórico-metodológicos para diseñar la planeación, requiere equilibrar lo que Kahneman señala como reparto de recursos atencionales para la tarea por atender; el mismo mecanismo evalúa la demanda de esfuerzo de la tarea. Este hallazgo representa una desvinculación entre la enseñanza por proyectos de varias asignaturas y los sustentos teóricos necesarios para desarrollarlos. Al atender estas actividades, se incrementa el índice lector de los alumnos y las producciones de textos no evidenciadas en los hallazgos; y en los docentes, la producción escrita, una planeación diseñada para cubrir los fines. Consideramos esencial mostrar los espacios del currículum que ofrecen posibilidades y condiciones de articulación a favor del aprendizaje, proponiendo el arousal en un nivel óptimo de activación:

- las líneas curriculares (el qué)

- el derecho a la educación de calidad en toda su extensión y posibilidad (el porqué)

- los fines de la educación en México (el para qué)

- el enfoque metodológico y las estrategias de enseñanza y aprendizaje (el cómo)

- los docentes en su papel mediador y los estudiantes en su rol proactivo (quiénes)

- las sesiones de clase de cada asignatura (cuándo, los tiempos de enseñanza)

- las aulas para el aprendizaje y espacios culturales de las escuelas (dónde)

Las limitaciones de la atención selectiva para la adquisición de los aprendizajes esperados en tanto aprendizaje de los alumnos — proponemos - residen en la ausencia de un repertorio lingüístico conceptual, disciplinario e interdisciplinario desde el cual otorgar significado y sentido a las experiencias y vivencias de aprendizaje de los alumnos, así como en las articulaciones metodológicas y curriculares para realizar el proceso de evaluación. La reducción de la evaluación se distingue por la desatención al aprendizaje individualizado y la ausencia de retroalimentación individual; en contraparte, se promueve la fijación y sedimentación del polo cuantitativo hecho norma en la acreditación. Se enfoca a la calificación que "sacó" un alumno, lo concreto se ofrece como una prueba; sin embargo, lo abstracto del proceso en la evaluación formativa está endeble.

En cambio, la atención individualizada sí existe para los alumnos con capacidades diferenciadas, como un producto y testimonio para la inclusión educativa; sin embargo, esta atención selectiva en la línea temporal de duración y continuidad resulta imposible, aunque es compensada con las adecuaciones curriculares; el profesor se asume como una parte de la solución.

Respecto a la evaluación, se concluye visible y concretamente que la atención selectiva puesta en las características de la competencia, los aprendizajes esperados, las evaluaciones diarias y semestrales, los cuadernos de los alumnos, la participación en el aula, el trabajo co- 
laborativo, es a lo que la atención del docente se acomoda más fácilmente y se realiza de mejor modo, como objetos de percepción. En esta investigación exploratoria, la atención, entendida como un foco de claridad, sí alcanza a distinguir arousal en las siguientes realidades: variados instrumentos y modos para evaluar, la especificidad de la evaluación cualitativa, la elaboración de rúbricas para aprendizajes, la particularidad de herramientas para la evaluación, la comprensión como desarrollo cognitivo. "Arousal", que remite al reconocimiento de dos núcleos temáticos favorecidos en las respuestas de los maestros y centrados en la relación evaluación y atención, se refiere a la relación entre la atención selectiva y los aspectos de la transferencia, principalmente centrados en contenidos y su adaptación evaluativa, cómo evaluar esta transferencia; el núcleo temático de la creación de herramientas prácticas para evaluar la aplicación del concepto de transversalidad. Si en la cuestión de la transferencia aplica la intervención de la atención teniendo por objeto condiciones subjetivas y epistémicas, en la cuestión de la transversalidad atañe a la diversidad disciplinaria de los objetos de conocimiento. La primera, de índole subjetiva; la segunda de índole objetiva. Ambas constituyen actividades procesuales y abstractas, así como exigen un producto creado. En esta situación de apertura hacia lo inédito, lo entendemos como la necesidad de crear un acto nuevo de evaluación de transferencia y transversalidad, una suspensión del habitus y, a la vez, la creación de una intervención de un significado del uso del tiempo psicológico de índole epistémico del profesor.

Reiteramos que la teoría está inscrita en conexión con la atención selectiva del profesor en tres campos articulados desde el aprendizaje, el primero alude al interés por la comprensión e interpretación, análisis, razonamiento y cognición en el campo de la abstracción, el segundo atiende a la evaluación y la coevaluación, el tercero remite al enfoque pedagógico y la planeación. El reto consiste en establecer las mediaciones específicas desde cada campo hacia el aprendizaje y está centrado en la experiencia del profesor con su conocimiento práctico desde el plan de estudios en educación básica.

Concluimos que la articulación entre la práctica y la teoría está en correspondencia con la variación de los tipos de atención; que de la práctica a la teoría existe un sendero de unificación que presenta la conexión entre aprendizajes esperados con el aprendizaje significativo; el programa de estudios, la distinción y claridad sobre las competencias, el enfoque pedagógico y la propuesta curricular.

La investigación muestra la escuela tradicional de enseñanza, aún presente en las prácticas docentes de planificación, aprendizaje y evaluación, con apenas un atisbo de metodología y referente teórico actual.

Reconocer la importancia de las condiciones atencionales está en relación con aquella recomendación de Perrenoud acerca de evitar la proletarización del profesor (2007). Es momento para el apego docente a los saberes legados por pedagogos de todos los tiempos, con función del nivel de activación o arousal, y de la capacidad específica que necesita cada actividad del 
proceso de enseñanza-aprendizaje. Entre otros saberes pedagógicos, se requiere del legado de Suchman para comprender la enseñanza por indagación y su trascendencia en la enseñanza por proyectos; del análisis de la escuela experimental de Dewey, a fin de reconsiderar al sujeto de la formación estudiantil en su faceta experimental y autoevaluable; del legado de Decroly, para manejar con propiedad los centros de interés, detonantes de proyectos de aprendizaje, detección de intereses y necesidades de atención de los estudiantes, incluso los de necesidades diferenciadas; del invaluable legado de Ausubel, el aprendizaje significativo que permea en alto porcentaje de las líneas curriculares (Plan de estudios 2011, Modelo educativo 2017); de la teoría de Bruner sobre el aprendizaje por descubrimiento; de los invaluables legados de Piaget y Vigostky, con el constructivismo genético de uno y el socioconstructivismo del otro. El eje de la didáctica para llegar a los fines de la educación se fortalece con estos legados como detonantes del proceso atencional a las líneas curriculares trascendentes. La formación profesional se fundamenta en ellos, con el compromiso de transformarse con pedagogía crítica, en y para las aulas.

La capacidad de esfuerzo y autorregulación cognitiva se asocian al proceso de reaprendizaje y a sus implicaciones en la planificación, buscando desde luego el nivel atencional óptimo de los profesores para la acción intencional de planificar la enseñanza y la evaluación. La nueva comprensión del docente requiere de procesos de reformulación del conocimiento práctico señalados por Shulman y Elbaz, y la reformulación de los conocimientos teórico en prácticos, señalados por Morin.

En términos ontológicos, la atención selectiva representa la seguridad de un asidero en una realidad compleja. La cuestión de la unicidad del aprendizaje, solicita o exige al profesor la aplicación de la diversidad de condiciones atencionales, y este encuentro con la realidad rebasa la atención selectiva como portadora de seguridad; para la enseñanza, es necesaria pero no suficiente. En un marco histórico y cultural de uso especial de la atención (libro verde), estas condiciones atencionales participan en procesos psicológicos, fenomenológicos, neurológicos y, en la situación de enseñanza-aprendizaje, en la experiencia presuponen una participación de la atención dirigida por la intencionalidad hacia la intersubjetividad, o bien, orientada a la realidad de los fenómenos objetivos o de los fenómenos subjetivos; corresponde al profesor vivir y elegir las rutas de orientación de los trabajos de las condiciones atencionales. El solo hecho de la intervención de un nuevo significado, un nuevo sentido en las dimensiones de la intersubjetividad, la objetividad o la subjetividad, requiere de una relaboración del sentido del tiempo. La planeación, la evaluación, valen como uso de tiempo; la intencionalidad y la orientación, así como la organización de ellas, valen como atención. En esta conexión como tipo de una realidad compleja entre atención y temporalidad ¿cómo puede comprenderse la enseñanza y, sobre todo, cómo la enseñanza puede comprender al aprendizaje? 
La relevancia científica de este reporte de investigación está en reconocer los retos cognitivos atendidos y logrados por los profesores y los retos cognitivos no atendidos por estos, en materia pedagógico-curricular y áulica; reconocemos que aún el docente puede, en cualquier momento, transformar la intensidad de atención que la tarea le representa, lograr el reto cognitivo conforme revise los elementos de interés y necesidad acordes a los objetivos; replantear la planificación responsablemente, indudable por el derecho de todos a la educación de calidad.

Hemos planteado la actividad docente como objeto de estudio en construcción, con sus procesos, condiciones y tipos atencionales. Esta construcción teórica da pauta para realizar un proceso de mediación acerca de los opuestos que sostiene la actividad docente, la relación planeación y tiempo profesional constituyen una promesa de investigación acción de gran interés en favor de algunos replanteamientos de la atención en la práctica docente.

\section{Referencias}

Alameda-Bailén, J. R. (2004). “Procesos de atención percepción y memoria”. En J. M. Mestre-Navas y F. Palmero-Cantero (coords). Procesos psicológicos básicos. España: McGraw-Hill Interamericana. http://www.uhu.es/jose.alameda/apm2006/tema1(06-07).pdf

Álvarez-Gayou Jurgenson, J. L. (2003). Cómo hacer investigación cualitativa: fundamentos y metodología. México: Paidós.

Añaños-Carrasco, E. (2002). Psicología de la atención y de la percepción. Barcelona: Universidad Autónoma de Barcelona.

Ballesteros, S. (2014). “La atención selectiva modula el procesamiento de la información y la memoria implícita [Selective attention modulates information processing and implicit memory]". Acción Psicológica, 11(1), 7-20. Madrid: Universidad Nacional de Educación a Distancia. http://dx.doi. org/10.5944/ap.1.1.13788

Depraz, N. (2012). Fenomenología de la atención "after"Husserl. Universidad de Rouen. Archivos Husserl. París: Centro de Investigación en Epistemología Aplicada (CREA). París Francia. Acta fenomenológica latinoamericana. Volumen IV (Actas del V Coloquio Latinoamericano de Fenomenología). Círculo Latinoamericano de Fenomenología Lima: Pontificia Universidad Católica del Perú, 21-40.

Domínguez, A. L. y J. Yáñez-Canal (2013). “El concepto de atención y consciencia en la obra de William James". Revista Colombiana de Psicología, 22(1), 199-214. Bogotá: Universidad Nacional de Colombia. http://www.redalyc.org/articulo.oa?id=80428081014

Fuenmayor, G. e Y.Villasmil (2008). “La percepción, la atención y la memoria como procesos cognitivos utilizados para la comprensión textual". Revista de Artes y Humanidades UNICA, 9(22), 187-202. Maracaibo: Universidad Católica Cecilio Acosta. 
Ison, M. S. y C. Korzeniowski (2016). “El rol de la atención y percepción viso-espacial en el desempeño lector en la mediana infancia". Psykhe, 25(1), 1-13. Santiago: Pontificia Universidad Católica de Chile. http://www.redalyc.org/articulo.oa?id=96745598007

James, W. (1890). Principios de psicología. Madrid: Daniel Jorro.

(1890). Sensación, percepción y atención. Universidad de Alicante. Psicología básica. http:// www.psb.ua.es

Kahneman, D. (1973). Attention and Effort. Nueva Jersey: Prentice-Hall.

(1997). Atención y esfuerzo. Madrid: Biblioteca Nueva.

López-López, L. I. (2015). “El conocimiento didáctico de los profesores de ciencias principiantes y experimentados en la enseñanza por competencias. Investigación con estudio de caso en educación secundaria". Tesis de Doctorado. Monterrey: Instituto para el Fomento científico de Monterrey (México).

Martínez-Bonafé, J. (2004). "La formación del profesorado y el discurso de las competencias". Profesorado. Revista Interuniversitaria de Formación del Profesorado, 18(3), 127-143.

Munar, E., J. Rosselló y A. Sánchez-Cabaco (coords.). (1999). Atención y percepción, selección para la acción. Madrid: Alianza Editorial.

Paquay, L. y M. C. Wagner (2005). “Formación continua y videoformación del maestro, qué habilidades se deben priorizar". En L. Paquay, M. Altet, E. Charlier y Ph. Perrenoud (coords.). La formación profesional del maestro. México: FCE, 222-264.

Pereira, F. (2015). “La atención y los límites de la experiencia consciente". Revista Filosofía Unísonos, 16(2), 145-163.

Perrenoud, P. (2004). Diez nuevas competencias para enseñar. Barcelona: Grao.

(2005). “El trabajo sobre el habitus en la formación de maestros. Análisis de las prácticas y toma de conciencia". En L. Paquay, M. Altet, E. Charlier y Ph. Perrenoud (coords.). La formación profesional del maestro. México: FCE, 265-308.

(2007). Desarrollar la práctica reflexiva en el oficio de enseñar. Barcelona: Grao.

Reales-Avilés, J. M. y S. Ballesteros-Jiménez (2000). "Atención y memoria implícita". Revista Anthropos: Huellas del Conocimiento, 150-159.

Ricoeur, P. (1939). "L'attention. Etude phénoménologique de l'attention et de ses connexions philosophiques". Bulletin du Cercle Philosophique de l'Ouest, 4 (1940), janvier-mars, 1-28 [polycopie].

Rodríguez, J. A. (2008). Cognición y ciencia cognitiva. Suecia: Universidad de Gotemburgo. http:// docplayer.es/23884754-Cognicion-y-ciencia-cognitiva-julio-alberto-rodriguez.html

Rodríguez, G., J. Flores y E. García (1999). Metodología de la investigación cualitativa. Granada: Ediciones Aljibe.

Rosselló, J. (1994). Psicología de la Atención. Manual introductorio al estudio del mecanismo atencional. España: Pirámide. https://www.academia.edu/1320393/Psicolog\%C3\%ADa de la atenci\%C3\%B3n introducci\%C3\%B3n al estudio del mecanismo atencional

Diálo pos 
Scheler, M. (1987). Esencia y formas de la simpatía. Buenos Aires: Losada.

Shulman, L. S. (1987). "El conocimiento didáctico del contenido (PCK)". Profesorado. Revista de Currículum y Formación del Profesorado. Granada: Universidad de Granada.

Stake, R. E. (2005). Investigación con estudio de casos. Madrid: Morata.

Tardif, M. y C. Gauthier (2005). "El maestro como 'actor racional': racionalidad, conocimiento, juicio". En La formación professional del maestro. México: FCE, 309-354.

Tejada-Fernández, J. (2009). "Competencias docentes". Profesorado. Revista de Currículum y Formación de Profesorado, 13( 2), 1-15. Granada: Universidad de Granada.

Tudela-Garmendia, P. (2015). Percepción y atención. España: Centro de Estudios Financieros, D.L. Villanueva-Gutiérrez, O. E. (2010). De estudiantes a profesores: transiciones y dilemas en la incorporación profesional. México: Editorial Porrúa.

SEP (2011). Plan y programas de estudio. Secundaria. México: SEP.

Waldenfels, B. (2015). Exploraciones fenomenológicas acerca de lo extraño. México: Anthropos, Siglo XXI. 